Research, Society and Development, v. 11, n. 2, e15511225500, 2022

(CC BY 4.0) | ISSN 2525-3409 | DOI: http://dx.doi.org/10.33448/rsd-v11i2.25500

\title{
Protease com atividade fibrinolítica e colagenolítica produzida por Aspergillus ochraceus URM604
}

\author{
Protease with fibrinolytic and collagenolytic activity produced by Aspergillus ochraceus URM604 \\ Proteasa con actividad fbrinolítica y colagenolítica producida por Aspergillus ochraceus URM604
}

Recebido: 08/01/2022 | Revisado: 15/01/2022 | Aceito: 19/01/2022 | Publicado: 22/01/2022

Kethylen Barbara Barbosa Cardoso ORCID: https://orcid.org/0000-0003-0317-0307

Universidade Federal de Pernambuco, Brasil E-mail: kethybarbara@gmail.com

Thiago Pajeú Nascimento

ORCID: https://orcid.org/0000-0003-3480-6734

Universidade Federal do Piauí, Brasil E-mail: thiago_pajeu@hotmail.com

Vagne de Melo Oliveira

ORCID: https://orcid.org/0000-0003-0841-1974 Universidade Federal do Acre, Brasil E-mail: vagne_melo@hotmail.com

Romero Marcos Pedrosa Brandão Costa

ORCID: https://orcid.org/0000-0001-7045-2975 Universidade de Pernambuco, Brasil

E-mail: romero_brandao@yahoo.com.br

Lorenzo Pastrana

ORCID: https://orcid.org/0000-0002-6637-3462 International Iberian Nanotechnology Laboratory, Portugal E-mail: lorenzo.pastrana@inl.int

Juanize Matias da Silva Batista

ORCID: http://orcid.org/0000-0001-7654-2533

Universidade Federal Rural de Pernambuco, Brasil E-mail: juanizematias@yahoo.com.br

Ana Lúcia Figueiredo Porto

ORCID: https://orcid.org/0000-0001-5561-5158

Universidade Federal Rural de Pernambuco, Brasil E-mail: analuporto@yahoo.com.br

\section{Resumo}

Proteases possuem um papel fundamental na economia, estando presentes em diversos setores industriais. Dentre os fungos conhecidos por produzir proteases, destaca-se o gênero Aspergillus. Desta forma, o objetivo deste trabalho foi produzir proteases a partir de fermentação em estado sólido de Aspergillus ochraceus, purificar, caracterizar e avaliar seu potencial na produção de enzima colagenolítica e fibrinolítica. Para isto foram avaliados o tipo e quantidade do substrato e a umidade das fermentações. Posteriormente foi realizada purificação por cromatografia DEA-Sephadex e Superdex-G75 em sistema FPLC. Assim como caracterização parcial avaliando o pH e temperatura ótimos da ação da protease. Foi observada que a melhor atividade da enzima nas fermentações com farelo de trigo como substrato (3g) e $60 \%$ de umidade, apresentando atividade proteásica, colagenolítica e fibrinolítica. Também foi observada maior atividade em $\mathrm{pH} 8$ e $50^{\circ} \mathrm{C}$. Assim, concluísse que A. ochraceus possui grande potencial para aplicação biotecnológica, destacando-se a importância de futuras avaliações.

Palavras-chave: Fungo Filamentoso; Resíduo de Café; Farelo de Trigo; Enzima Fibrinolítica; Colagenase.

\begin{abstract}
Proteases play a fundamental role in the economy, being present in several industrial sectors. Among the fungi known to produce proteases, the genus Aspergillus stands out. Thus, the objective of this work was to produce proteases from solid state fermentation of Aspergillus ochraceus, purify, characterize and evaluate their potential in the production of collagenolytic and fibrinolytic enzymes. For this, the type and quantity of the substrate and the humidity of the fermentations were evaluated. Subsequently, purification was performed by DEA-Sephadex and Superdex-G75 chromatography in an FPLC system. As well as partial characterization evaluating the optimal $\mathrm{pH}$ and temperature of the protease action. It was observed that the best enzyme activity in fermentations with wheat bran as substrate (3g) and $60 \%$ moisture, showing protease, collagenolytic and fibrinolytic activity. Greater activity was also observed at $\mathrm{pH} 8$ and
\end{abstract}


$50^{\circ} \mathrm{C}$. Thus, it was concluded that A. ochraceus has great potential for biotechnological application, highlighting the importance of future evaluations.

Keywords: Filamentous Fungus; Coffee Residue; Wheat Bran; Fibrinolytic Enzyme; Collagenase.

\begin{abstract}
Resumen
Las proteasas juegan un papel fundamental en la economía, estando presente en varios sectores industriales. Entre los hongos que se sabe que producen proteasas, se destaca el género Aspergillus. Así, el objetivo de este trabajo fue producir proteasas a partir de la fermentación en estado sólido de Aspergillus ochraceus, purificar, caracterizar y evaluar su potencial en la producción de enzimas colagenolíticas y fibrinolíticas. Para ello se evaluó el tipo y cantidad de sustrato y la humedad de las fermentaciones. Posteriormente, se realizó la purificación mediante cromatografía DEASephadex y Superdex-G75 en sistema FPLC. Así como la caracterización parcial evaluando el pH y la temperatura óptimos de la acción de la proteasa. Se observó que la mejor actividad enzimática en fermentaciones con salvado de trigo como sustrato $(3 \mathrm{~g})$ y $60 \%$ de humedad, presenta actividad proteasa, colagenolítica y fibrinolítica. También se observó una mayor actividad a pH 8 y $50{ }^{\circ} \mathrm{C}$. Así, se concluyó que A. ochraceus tiene un gran potencial de aplicación biotecnológica, destacando la importancia de futuras evaluaciones.
\end{abstract}

Palabras clave: Hongo Filamentoso; Residuo de Café; Salvado de Trigo; Enzima Fibrinolítica; Colagenasa.

\title{
1. Introdução
}

Proteases são biocatalisadores valiosos, amplamente empregadas em diversos setores industriais principalmente devido a sua diversidade bioquímica, facilidade quanto de manipulação e especificidade (Clerici et al., 2021). Proteases com capacidade de degradar o colágeno, por exemplo, podem ser utilizadas na indústria do couro, alimentícia e farmacêutica (Bhagwat \& Dandge 2018). Além disso, peptídeos de colágeno ainda podem ser empregados na fabricação de fármacos, hidratantes, suplementos, agentes antimicrobianos, entre outros (Yagoda et al., 2014). Outro destaque no mercado de enzimas são as proteases com atividade fibrinolítica, uma vez que apresentam dissolução de coágulos sem alteração do fluxo sanguíneo, podendo ser utilizadas para tratamento terapêutico da trombose (Ali \& Bavisetty, 2020; Nascimento et al., 2015).

A partir da importância dessas enzimas, a diversidade microbiana é explorada na finalidade de identificar-se microrganismos produtores de protease, uma vez que estes destacam-se metabólitos (Clerici et al., 2021). Proteases produzidas por fungos filamentosos destacam-se ainda em relação a sua atividade (Kilikian \& Pessoa-Jr, 2020; Wanderley et al. 2017). Dentre as espécies mais utilizadas na produção de enzimas encontram-se as pertencentes ao gênero Aspergillus, como A. niger, A. fumigatus, e A. oryzae, conhecidos por secretar uma gama de enzimas e proteínas extracelulares que podem ser utilizadas para diversos processos industriais (Bianco, L. \& Perrotta, G., 2015), entretanto existe uma grande variedade de espécies ainda não estudadas. Segundo El-Khonezy et al. (2021) Aspergillus ochraceus BT21 foi capaz de produzir protease alcalina, apresentado estabilidade com detergentes comerciais. Outros estudos destacam ainda o potencial colagenolítico de proteases produzidas por A. ochraeus, entretanto as informações sobre o uso deste microrganismo para fabricação enzimática são escassas.

Segundo a literatura, a produção de enzimas por fermentação em estado sólido (FES) é promissora (Novelli et al., 2016). Uma das principais vantagens dessa metodologia é a possibilidade do uso de resíduos orgânicos, como cascas de laranja, maçã, banana, farelo de soja, arroz e trigo, sendo este último um dos substratos mais descritos na literatura para fermentação microbiológica (Chimbekujwo, et al. 2013) outros resíduos menos comuns também demonstram ser substratos relevantes, como a borra de café (Pandey et al., 2000; Rocha et al., 2021). Além disso, utiliza pouca quantidade de água o que promove uma maior concentração de metabólitos, reduz a probabilidade de contaminação bacteriana e favorece o crescimento de fungos filamentosos, já que estes crescem facilmente sobre substratos sólidos (Chilakamarry et al., 2022). Por tanto, o objetivo deste trabalho é produzir e purificar proteases de interesse comercial a partir da fermentação em estado sólido de Aspergillus ochraceus URM604, bem como avaliar a utilização de farelo de trigo e resíduos de café como substratos neste bioprocesso. 


\section{Metodologia}

\subsection{Microrganismo}

O fungo foi cedido em parceria com o Departamento de Micologia da Universidade Federal de Pernambuco Micoteca URM604. O meio utilizado para a manutenção dos micro-organismos foi o Ágar Batata Dextrose (BDA) previamente autoclavado a $121^{\circ} \mathrm{C}$ por $20 \mathrm{~min}$ (Nascimento et al. 2017).

\subsection{Fermentação em Estado Sólido (FES)}

A FES foi determinada segundo Rocha et al. (2021), adaptado. O meio de cultura para a produção das proteases foi obtido a partir de comércio local, sendo coletado o farelo de trigo e a borra de café, estes foram previamente secos em estufa a $100^{\circ} \mathrm{C}$ até a completa desidratação e peneirados. Foi realizado planejamento fatorial $2^{3}$ (Tabela 1) para análise das melhores condições de produção, onde foram avaliados quanto ao tipo de substrato (café, trigo, e café + trigo em uma mistura de 1:1), à concentração de substrato $(3 \mathrm{~g}, 5 \mathrm{~g}$ e $7 \mathrm{~g})$ e umidade $\left(20 \%, 40 \%\right.$ e 60\%). Para a FES, os substratos foram autoclavados a $121^{\circ} \mathrm{C}$ por $20 \mathrm{~min}$, em erlenmeyers de $125 \mathrm{~mL}$. Posteriormente foram depositados nestes erlenmeyers os esporos do fungo na concentração $10^{7}$, ressuspendidos em solução de com caldo glicosado ( $1 \%$ Glu, $0,2 \%$ extrato de carne e $3 \%$ peptona) e tween 80 (w/n). A fermentação ocorreu por 7 dias em estufa a $30^{\circ} \mathrm{C}$, sem luminosidade.

Tabela 1: Matrix do planejamento fatorial $2^{3}$

\begin{tabular}{cccc}
\hline \multicolumn{1}{c}{ Variáveis } & \multicolumn{3}{c}{ Níveis } \\
\cline { 2 - 4 } & Menor (-1) & Central (0) & Maior (+1) \\
\hline $\begin{array}{c}\text { Concentração do } \\
\text { substrato (g) }\end{array}$ & 3 & 5 & \\
\hline Umidade (\%) & 20 & 40 & 60 \\
\hline Tipo do substrato & Café & Café + trigo & Trigo \\
\hline
\end{tabular}

Fonte: Autores (2022)

\subsection{Extração da Enzima e Obtenção de precipitado cetônico}

A extração da enzima foi realizada segundo Rocha et al. (2021), utilizando Tampão TRIS-HCl pH $80,1 \mathrm{M}$ com NaCl a 0,15M (7 ml/g de substrato). A filtração para obtenção do líquido metabólico foi realizada em bomba a vácuo. Para obtenção do precipitado cetônico, uma alíquota do extrato bruto $(10 \mathrm{~mL})$ foi precipitado com acetona $(70 \%)$ e solubilizado em $1 \mathrm{~mL}$ do mesmo tampão (Nascimento et al. 2017).

\subsection{Dosagem Proteica e Atividade Proteolítica}

A determinação das proteínas totais foi realizada utilizando o método BCA de acordo com Smith et al. (1985). O método para determinação da atividade da protease foi descrito por Ginther (1979) modificado. A mistura de reação conteve $0,25 \mathrm{~mL}$ do substrato (azocaseína 1\% p/v em tampão Tris- $\mathrm{HCl}$ 0,1M, pH 8,0) e 0,15 mL da amostra. Esta mistura foi incubada por 1 hora em estufa à $30^{\circ} \mathrm{C}$ sendo a reação interrompida pela adição de $1 \mathrm{~mL}$ de ácido tricloroacético (TCA) $10 \%$ p/v., posteriormente as amostras foram centrifugadas por 5 minutos a $10000 \mathrm{rpm}$. Dos sobrenadantes, foram pipetados $0,8 \mathrm{~mL} \mathrm{e}$ 
transferidos para eppendorfs contendo $0,2 \mathrm{~mL}$ de hidróxido de sódio 1,8 $\mathrm{N}$. A leitura foi realizada a 420nm em espectrofotômetro.

\subsection{Atividades Colagenolítica e Fibrinolítica}

A atividade colagenolítica foi determinada de acordo com o método de Chavira et al. (1984), modificado. Uma suspensão de Azocoll a $5 \mathrm{mg} / \mathrm{mL}$ foi preparada em tampão 0.1M Tris- $\mathrm{HCl}$, pH 8,0 para um volume final de 0,950 mL, $0,050 \mathrm{~mL}$ da amostra foi inoculada nessa suspensão e a reação processada a $35^{\circ} \mathrm{C}$, por 1 hora sob agitação. Em seguida, a reação foi centrifugada 5000 rpm por 5min, e a absorbância medida em 520nm em espectrofotômetro. A Atividade específica de ambos os experimentos foi determinada através da razão Atividade/proteína e expressa em U/mg.

A atividade fibrinolítica foi determinada de acordo com Wang et al. (2006). Para formação do coágulo foi utilizado $0,4 \mathrm{~mL}$ de fibrinogênio $0,72 \%$ com $0,1 \mathrm{~mL}$ de tampão fosfato $245 \mathrm{mM}\left(\mathrm{pH} 7\right.$ ) e incubado a $37^{\circ} \mathrm{C}$ por $5 \mathrm{~min}$. Em seguida, foi adicionado $0,1 \mathrm{~mL}$ de uma solução de trombina $20 \mathrm{U} / \mathrm{mL}$. A solução foi incubada a $37^{\circ} \mathrm{C}$ por 10 min. Posteriormente foi adicionado $0,1 \mathrm{~mL}$ de amostra contendo a enzima e a incubação continuou a $37^{\circ} \mathrm{C}$. A solução sofreu agitação para homogeneização a cada 20 minutos até completar 1 hora. Por fim, foi adicionado $0,7 \mathrm{~mL}$ de ácido tricloroacético $0,2 \mathrm{M}$ (TCA) para parar a reação. A mistura de reação foi centrifugada a 15.000 x g durante $10 \mathrm{~min}$. Então, $1 \mathrm{~mL}$ do sobrenadante foi recolhido e a absorbância a 275nm foi medida. A atividade foi medida levando em consideração que 1 unidade (unidade de degradação de fibrina, FU) de a atividade enzimática é definida como um aumento de 0,01 por minuto na absorbância a 275 nm da solução de reação.

\subsection{Purificação da enzima através de métodos cromatográficos}

O método de purificação das enzimas foi realizado utilizando cromatografia de troca iônica em DEAE-sephadex de acordo com Nascimento et al. (2017), a amostra foi eluída com solução salina de $\mathrm{NaCl}$ a $0,3 \mathrm{M}$ com tampão TRIS-HCl pH 8, 0,1M. Em seguida, a enzima passou por sistema FPLC através do equipamento Akta Avant 25 onde foi utilizada coluna cromatográfica em gel filtração, Superdex-G75.

\subsection{Caracterização bioquímica parcial}

\subsubsection{Efeito do pH e Temperatura na Atividade Enzimática}

O efeito do $\mathrm{pH}$ na atividade da protease foi avaliado pela mistura da solução enzimática com o substrato específico, preparados em soluções tampões $0,05 \mathrm{M}$ com diferentes valores de $\mathrm{pH}$ : citrato de sódio (pH 5,8; 6,2; 7,2); fosfato de sódio (pH 5,8; 6,2); Tris- $\mathrm{HCl}(\mathrm{pH} 7,2 ; 8,6$ e 9,0) e Glicina-NaOH (pH 8,6, 9,0 e 9,5) e determinações da atividade enzimática. O efeito da temperatura foi determinado utilizando uma mistura de reação contendo o substrato específico e a solução enzimática, incubadas em diferentes temperaturas $\left(25^{\circ} \mathrm{C}, 30^{\circ} \mathrm{C}, 40^{\circ} \mathrm{C}, 50^{\circ} \mathrm{C}, 60^{\circ} \mathrm{C}\right.$ e $\left.70^{\circ} \mathrm{C}\right)$ por 60 min, para posterior determinação da atividade enzimática (Nascimento et al. 2017).

\section{Resultados e Discussão}

Fatores como umidade, tipo e concentração do substrato foram estudados visando otimizar a produção de protease. Todas as culturas de Aspergillus ochraceus URM604 foram capazes de crescer nas condições avaliadas, da mesma forma todos os extratos apresentaram um alto teor de proteínas e proteases. Estes substratos já foram utilizados por vários autores, o farelo de trigo é frequentemente relatado na literatura para produção de enzimas, Nascimento et al. (2017) apresenta farelo de trigo como melhor substrato na produção de proteases com ação fibrinolítica a partir da fermentação em estado sólido com fungos 
filamentosos; Naik et al. (2019) obteve o mesmo resultado ao comparar farelo de trigo com resíduos agroindustriais na produção de enzimas por Aspergillus sp. O uso de resíduos de café é pouco mencionado na literatura embora apresente grande potencial (Cerda, et al., 2017), ainda assim foram utilizados em estudos para produção de celulases e xilanases por bactérias (Pseudoxanthonomas taiwanensis e Sphingobacterium composti) e leveduras (Cyberlindnera jardinii e Barnettozyma californica), demonstrando serem excelentes meios para o cultivo de microrganismos (Soccol et al., 2017). Rocha et al. (2021) utiliza resíduo de café para produção de proteases por Aspergillus sydowii URM5774, obtendo 59 U/mg em extrato bruto.

A relação entre as variáveis utilizadas e a proteases por Aspergillus ochraceus URM604 pode ser analisada na tabela 2, onde observa-se uma superioridade na atividade específica $(218,27 \mathrm{U} / \mathrm{mg}$ ) utilizando trigo (3g à $60 \%$ de umidade) quando comparado à borra de café pura $(76,44 \mathrm{U} / \mathrm{mg})$ nas mesmas condições de fermentação, apresentando ser o melhor substrato para obtenção destas enzimas. Também foram realizados testes com ambos os substratos na concentração de 1:1, nas condições centrais de $5 \mathrm{~g}$ de substrato e $40 \%$ de umidade, ainda assim, os resultados obtidos quando utilizado somente o farelo de trigo se mostraram superiores. Similarmente, El-Khonezy et al. (2021), obteve até 7359,8 U/mg utilizando farelo de trigo como substrato na produção de proteases por A. ochraceus em estudo onde foram avaliadas fermentações com milho íngreme, linho, farelo de trigo, farelo de arroz, feno e soja.

Tabela 2. Resultados da produção de protease por Aspergillus utilizando planejamento fatorial $2^{3}$

\begin{tabular}{ccccc}
\hline Substrato & Quantidade $(\mathrm{g})$ & Umidade $(\%)$ & Proteína Total $(\mathrm{mg} / \mathrm{mL})$ & $\begin{array}{c}\text { Atividade Proteásica } \\
\text { Específica }(\mathrm{U} / \mathrm{mg})\end{array}$ \\
\hline CAFÉ & 3 & 20 & 66,95 & 97,28 \\
CAFÉ & 3 & 60 & 89,05 & 76,44 \\
CAFÉ & 7 & 20 & 100,27 & 80,52 \\
CAFÉ & 7 & 60 & 107,50 & 65,58 \\
TRIGO & 3 & 20 & 44,75 & 182,19 \\
TRIGO & 3 & 60 & 31,55 & 218,27 \\
TRIGO & 7 & 20 & 42,16 & 139,71 \\
TRIGO & 7 & 60 & 35,08 & 207,07 \\
C+T* & 5 & 40 & 48,30 & 191,99 \\
\hline
\end{tabular}

$* \mathrm{C}+\mathrm{T}=50 \%$ de Borra de Café $+50 \%$ de Farelo de Trigo. Fonte: Autores (2022)

Em relação a umidade, a fermentação utilizando farelo de trigo como substrato apresenta maiores resultados em altas concentrações de umidade $(60 \%)$, em contrapartida, o resultado obtido a partir da FES com borra de café foi o oposto, as maiores atividades proteásica apresentam com as menores concentrações de umidade (20\%), estes resultados devem-se às diferenças de capacidade de absorção entre os substratos. Já a quantidade de substrato não afetou demasiadamente nenhuma das fermentações. Os resultados do estudo corroboram com a literatura, demostrando o potencial do gênero Aspergillus como produtor de protease, além de enfatizar o farelo de trigo como excelente substrato em fermentações em estado sólido (Soccol et al., 2017; Chilakamarry et al., 2022). 
Atividades específicas foram conduzidas para avaliar o potencial do microrganismo estudado na produção de enzimas com atividade fibrinolítica e colagenolíticas, tendo em vista que um mesmo microrganismo pode produzir diferentes biomoléculas. As avaliações foram realizadas ainda com o extrato bruto e os resultados são promissores, em relação a atividade colagenolítica foi possível observar 47,33 U/ml, já a atividade fibrinolítica foi expressa em 21,78 U/ml. Osmolovskiy et al. (2016) ao realizar estudo avaliativo do potencial de fungos filamentosos na produção de enzimas fibrinolíticas obteve 17.6 UTyr/mL fermentando do fungo A. ochraceus em meio líquido com pH 8. O tipo de fermentação utilizada interfere diretamente na concentração das proteínas obtidas, a quantidade de água livre presente na fermentação submersa pode diluir as proteases produzidas acarretando um menor rendimento. Em contraste, Nascimento et al. (2020) avalia a produção de enzima fibrinolítica por Mucor subtilissimus UCP1262 a partir de fermentação em estado sólido, obtendo resultado de até 26.1 U/ml.

A aplicação de uma protease na indústria, e mesmo a sua purificação, tem que ser pensada a partir das características físico-químicas apresentadas por estas biomoléculas. A avaliação da melhor temperatura e pH para atividade destas enzimas é um fator determinante para seu uso industrial. Para isto foi avaliado o $\mathrm{pH}$ ótimo e temperatura ótima das proteases produzidas por A. ochraceus URM604 (Figura 1), como pode ser observado, os melhores resultados foram obtidos em atividades pH 8 e temperatura de $50^{\circ} \mathrm{C}$. Em trabalho realizado com A. oryzae e A. flavipes, atividade proteásica manteve-se estável até $50^{\circ} \mathrm{C}$ (Novelli et al., 2016). Em concordância, a protease produzida por A. sidowii apresentou estabilidade térmica entre $25^{\circ} \mathrm{C}$ e $55^{\circ} \mathrm{C}$, e máxima atividade a $45^{\circ} \mathrm{C}$ (Rocha et al., 2020) $\mathrm{O}$ declínio nas atividades observadas neste trabalho e nos acima citados ocorrem pela desnaturação da enzima frente a altas temperaturas. Em relação ao pH ótimo, Amaral et al. (2020) defendem que proteases com propriedades alcalinas podem ser usadas na fabricação de couro, detergentes e indústria farmacêutica, estes apresentaram resultados similares ao obtidos neste trabalho ao fermentar Aspergillus tamarii Kita UCP1279.

Figura 1. pH ótimo [A] e Temperatura ótima [B] de protease produzida por Aspergillus ochraceus.

[A]

\section{[B]}

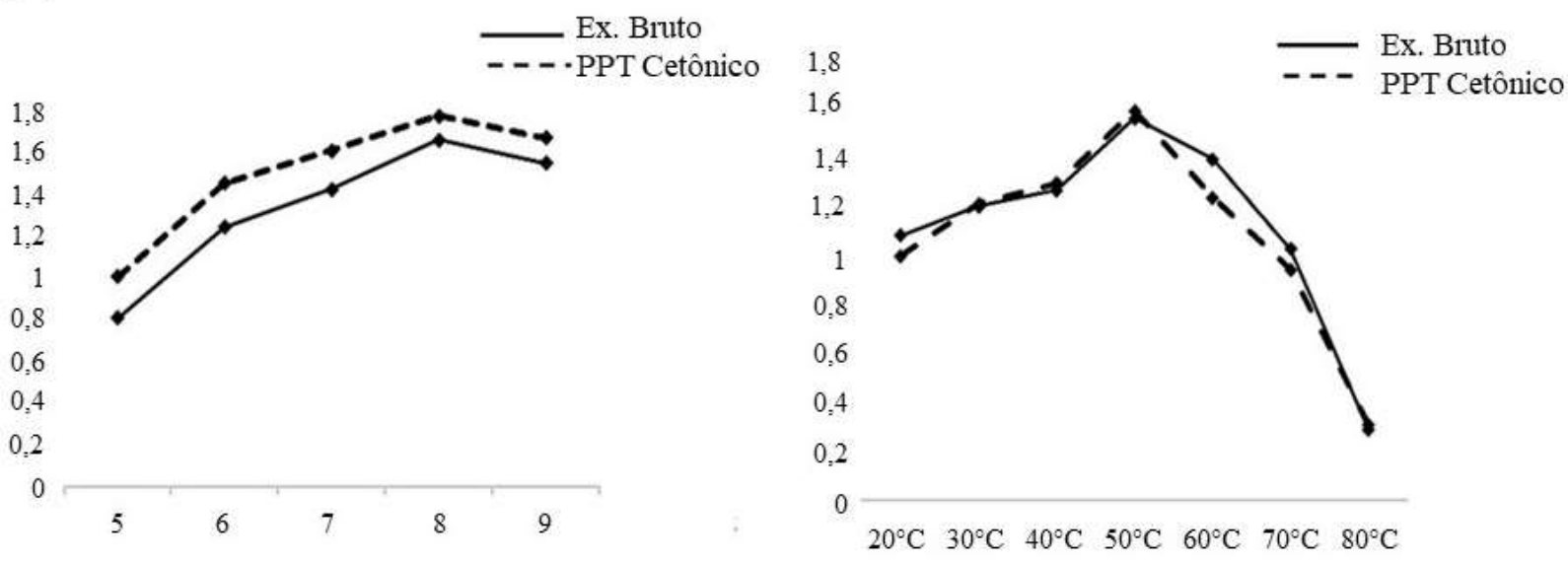

*Ex. Bruto = Extrato bruto. PPT Cetônico = Precipitado cetônico. Fonte: Autores (2022).

Os processos de purificação utilizados mostraram-se eficientes, obtendo-se uma recuperação de até 84,7\% (436,67 $\mathrm{U} / \mathrm{ml}$ ) nas frações eluídas com $0,3 \mathrm{M}$ de $\mathrm{NaCl}$ coletadas na coluna cromatográfica DEAE-Sephadex G50. Além disso, os picos ativos foram concentrados e depois submetidos a uma coluna Superdex G-75, obtendo-se um único pico ativo com atividade de 186,66 U/ml (Figura 2). 
Research, Society and Development, v. 11, n. 2, e15511225500, 2022

(CC BY 4.0) | ISSN 2525-3409 | DOI: http://dx.doi.org/10.33448/rsd-v11i2.25500

Figura 2. Perfil de eluição obtido no sistema Superdex-75 FPLC e pico com atividade proteásica.

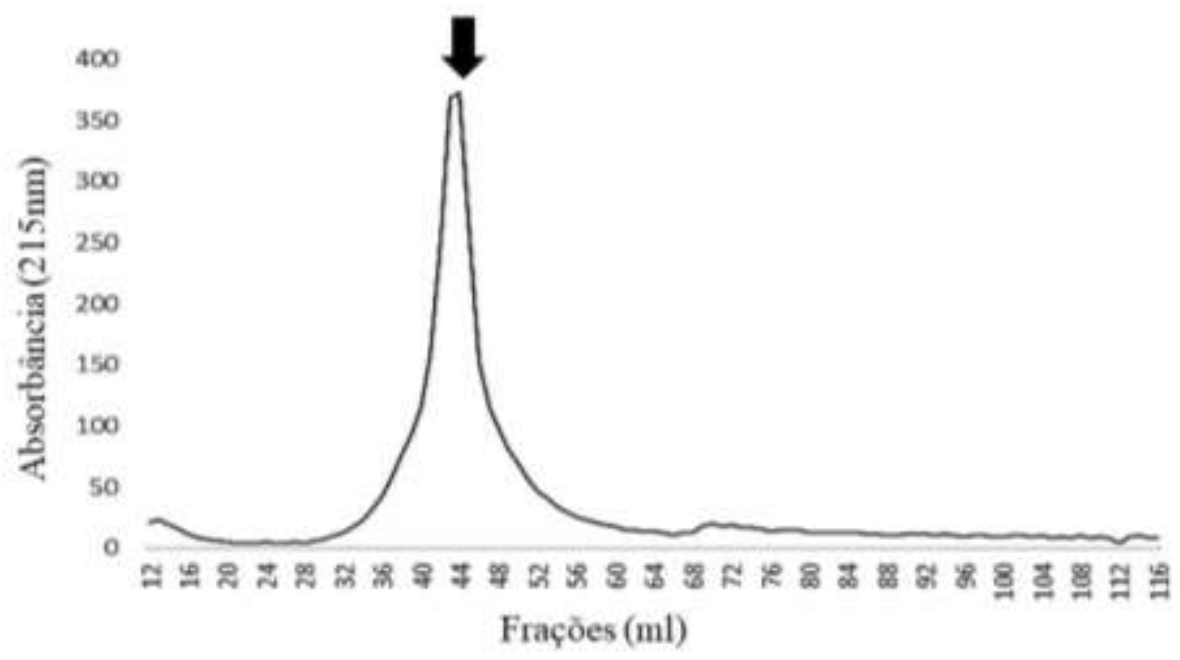

Fonte: Autores (2022).

\section{Conclusão}

O uso de resíduos agroindustriais para fermentação em estado sólido é eficaz, entretanto, apesar de ambos os substratos de borra de café e farelo de trigo apresentaram potencial para este viés, dentre as variáveis avaliadas a melhor condição para produção de proteases por Aspergillus ochraceus URM604, foram o uso do farelo de trigo puro, com $60 \%$ de umidade e $3 \mathrm{~g}$ de substrato. A protease obtida por Aspergillus ochraceus URM604 foi purificada por técnicas cromatográficas e apresenta potencial biotecnológico para uso na indústria de detergentes, amaciamento de couro e na indústria farmacêutica.

Os resultados obtidos no presente trabalho também demonstram o potencial deste microrganismo como produtor de protease com ação colagenolítica e fibrinolítica, fazendo-se necessário maiores estudos referentes a caracterização da protease e estudos de aplicação.

\section{Referências}

Ali, A. M. M.; \& Bavisetty, S. C. B. (2020). Purification, physicochemical properties, and statistical optimization of fibrinolytic enzymes especially from fermented foods: A comprehensive review. International Journal of Biological Macromolecules, 163(20), $1498-1517$.

Amaral, Y. M. S., Silva, A. O. S., Oliveira, R. L., \& Porto, T. S. (2020). Production, extraction, and thermodynamics protease partitioning from Aspergillus tamarii Kita UCP1279 using PEG/sodium citrate aqueous two-phase systems. Preparative Biochemistry \& Biotechnology, 1(20), 619-626.

Bhagwat, P. K \& Dandge, P. B. (2018). Collagen and collagenolytic proteases: A review. Biocatalysis and Agricultural Biotechnology. 15 (18), $43-55$.

Bianco, L. \& Perrotta, G. (2015) Methodologies and perspectives of proteomics applied to filamentous fungi: From sample preparation to secretome analysis. International Journal of Molecular Sciences. 16 (3), 5803-5829.

Cerda, A., Mejías, L., Gea, T., \& Sánchez, A. (2017). Cellulase and xylanase production at pilot scale by solid-state fermentation from coffee husk using specialized consortia: The consistency of the process and the microbial communities involved. Bioresource Technology. $243,1059-1068$.

Chavira, R. J.; Burnett, T. J.; \& Hageman, J. H. (1984). Assaying proteinases with Azocoll. Annalytical Biochemistry. 136, 4446-4450.

Chilakamarry, C. R., Sakinah, A. M. M., Zularisam, A. W., Sirohi, R., Khilji, I. A., Ahmad, N., \& Pandey, A. (2022) Advances in solid-state fermentation for bioconversion of agricultural wastes to value-added products: Opportunities and challenges. Bioresource Technology. 343(2022), 1-11.

Chimbekujwo, K. I., Ja'afaru, M. I., \& Adeyemo, O. M. (2020). Purification, characterization and optimization conditions of protease produced by Aspergillus brasiliensis strain BCW2. Scientific African. 8 (20), 1-9.

Clerici, N. J., Lermen, A. M., \& Freitas, D. J. D. (2021). Agro-industrial by-products as substrates for the production of bacterial protease and antioxidant hydrolysates. Biocatalysis and Agricultural Biotechnology. 37 (2021), 1-11. 
Research, Society and Development, v. 11, n. 2, e15511225500, 2022

(CC BY 4.0) | ISSN 2525-3409 | DOI: http://dx.doi.org/10.33448/rsd-v11i2.25500

El-Khonezy, M. Elgammal, E. W., Ahmed, E. F., \& Abd-Elaziz, A. M. (2021) Detergentstable thiol-dependant alkaline protease produced from the endophytic fungus Aspergillus ochraceus BT21: Purification and kinetics. Biocatalysis and Agricultural Biotechnology. 35 (2021), 1-9.

Ginther, C. L. (1979). Sporulation and the Production of Serine Protease and Cephamycin C by Streptomyces lactamdurans. Antimicrobial Agents and Chemotherapy. 15, 522-526.

Gurumallesh, P., Alagu, K., Ramakrishnan, B., \& Muthusamy, A.(2019). A systematic reconsideration on proteases. International Journal of Biological Macromolecules. 128, 254-257.

Naik, B., Goyala, S. K., Tripathi, A. D., \& Kumarc, V. (2019). Screening of agro-industrial waste and physical factors for the optimum production of pullulanase in solid-state fermentation from endophytic Aspergillus sp. Biocatalysis and Agricultural Biotechnology. 22 (19), 1-7.

Nascimento, T. P. Sales, A. E., Porto, C. S., Brandão, R. M. P., Takaki, G. M. C., Teixeira, J. A. C., Porto, T. S., \& Porto, A. L. F. (2015). Production and Characterization of New Fibrinolytic Protease from Mucor subtillissimus UCP 1262 in Solid-State Fermentation. Advances in Enzyme Research. 3 (3), 81-91.

Nascimento, T. P., . Sales, A. E., Porto, C. S., Brandão, R. M. P., Breydo, L., Uversky, V. N., Porto, A. L. F., \& Converti, A. (2017). Purification, biochemical, and structural characterization of a novel fibrinolytic enzyme from Mucor subtilissimus UCP 1262. Bioprocess and Biosystems Engineering, 40(8), 1209-1219.

Novelli, P.K, Barros, M.M, \& Fleuri, L.F. (2016). Novel inexpensive fungi proteases: Production by solid state fermentation and characterization, Food Chem. 198, 119-124.

Osmolovskiy, A. A., Popova, E. A., Kreyer, V. G., \& Baranová, N. (2016). Fibrinolytic and collagenolytic activity of extracellular proteinases of the strains of micromycetes Aspergillus ochraceus L-1 and Aspergillus ustus 1. Moscow University Biological Sciences Bulletin. 71, 62-66.

Pandey, A., Soccol, C. R., Nigam, P., Brand, D., Mohan, R., \& Roussos, S. (2000). Biotechnological potential of coffee pulp and coffee husk for bioprocesses. Biochemical Engineering Journal. 6(1), 153-162.

Pessoa-Jr, A. et al. Rompimento celular. In. Kilikian, B. V., \& Pessoa-jr, A. (2020) Purificação de produtos biotecnológicos: operações e processos com aplicação industrial. $2^{\mathrm{a}}$ ed. São Paulo: Blucher. 67-103.

Rocha, F. T. B., Brandão-costa, R. M. P., Neves, A. G. D., Cardoso, K. B. B., Nascimento, T. P., Albuquerque, W. W. C., \& Porto, A. L. F. (2021) Purification and characterization of a protease from Aspergillus sydowii URM5774: Coffee ground residue for protease production by solid state fermentation. Microbiology • An. Acad. Bras. Cienc. 93 (3), 1-13.

Smith, P.K.; Krohn, R.I.; Hermanson, G.T.; Mallia, A.K.; Gartner, F.H.; Provenzano, M.D.; Fujimoto, E.K.; Goeke, N.M.; Olson, B.J.; \& Klenk, D.C. (1985). Measurement of protein using bicinchoninic acid. Analytical Biochemistry. 150 (1), 76-85.

Soccol, C. R.; Costa, E. S. F.; Letti, L. A. J.; Karp, S. G.; Woiciechowski, A. L.; \& Vandenberghe, L. P. de S. (2017). Recent developments and innovations in solid state fermentation. Biotechnology Research and Innovation. 1, 52-71.

Tripathi, P., Tomar, R., \& Jagannadham, M. V. (2011). Purification and biochemical characterization of a novel protease streblin. Food Chemistry, 125, $1005-1012$

Wanderley, M. C. A., Duarte, J. M. W., Filho, J. L. L., Lima, C. A., Teixeira, J. A. C., \& Porto, A. L. F. (2017). Collagenolytic enzymes produced by fungi: a systematic review. Brazilian Journal of Microbiology. 48 (1), 1-13.

Wang, S. L., \& Yeh, P.Y. (2006). Production of a surfactant-and solvent stable alkaliphilic protease by bioconversion of shrimp shell wastes fermented by Bacillus subtilis TKU007. Process Biochem 41, 1545-1552. 